\title{
Design and Simulation of Array Lens for coupling of A 4×4 Nano-Scale VCSEL Array to A Single Mode Fiber
}

\author{
${ }^{1}$ Wen-Ming Cheng, ${ }^{1}$ Hen-Wai Tsao and ${ }^{2}$ Shyh-Lin Tsao \\ ${ }^{1}$ Graduate Institute of Electro-Optical Engineering, National Taiwan University, \\ No.1, Roosevelt Rd,. Sec. 4, Taipei, Taiwan 106, R.O.C. \\ ${ }^{2}$ Institute of Electro-Optical Science and Technology, National Taiwan Normal University, \\ No.88, Ting-Choui Rd,. Sec. 4, Taipei, Taiwan 116, R.O.C. \\ ${ }^{2}$ E-mail : jimmyslt@cc.ntnu.edu.tw \\ Apr. 29, 2004
}

\section{Introduction}

For realization of the high data rate transmission, the development of Vertical Cavity Surface Emitting Laser (VCSEL) array had boomed during the late ten years [1]. A single-crystal nanowire cannot only acts as an efficient electrically driven laser integrate into silicon-based systems but also as a single mode optical waveguide like an optical fiber $[2,3]$. Very small aperture lasers, with high optical power, are attractive high-speed sources for optical communication and data storage [4]. However, the research on the nano-scale VCSEL array still have an important issue, that is, the light coupling from VCSEL array to a single mode fiber (SMF) for increasing optical power coupling efficiency and brightness in micro-optical system. Based on Zemax optical design software with non-sequential and geometrical optics approximations [5], we propose a solution using microlens arrays for highly optical power coupling efficiency between $4 \times 4$ VCSEL array and a SMF to achieve the high speed data transfer of optical power division multiplexing in an optical fiber in this paper.

Applications of our designed microlens arrays, it is a useful solution for micro-optic design for more flexible alignment between optical fiber and nanowire laser arrays in nano-optical systems.

\section{Results and discussion}

The overall size of our considered $4 \times 4$ nano-scale VCSEL array is setup as $8 \mu \mathrm{m}^{2}$ with $2 \mu \mathrm{m}$ core pitch and $850 \mathrm{~nm}$ wavelength. Fig.1 shows the schematic diagram of the array lens for propagating of 16 elements of VCSEL in a SMF. The distance from VCSEL array to microlens is set as 640nm. The input end of the SMF is placed at $890 \mathrm{~nm}$ far from microlens output end. The detector is set as $10 \mu \mathrm{m}^{2}$ behind $1000 \mathrm{~mm}$ SMF. The core pitch between lenses is also $2 \mu \mathrm{m}$, matching the arrangement of our proposed $4 \times 4$ nano-scale VCSEL array. The core diameter of fiber of $\mathrm{Si}$ is $10 \mu \mathrm{m}$ and the cladding diameter of fiber is $125 \mu \mathrm{m}$.

We simulate and analyze our proposed system for multi-elements optical power coupling from VCSEL array to a SMF. In Fig. 2, we can find the transmission loss of our proposed array lens is from $0 \mathrm{~dB}$ to $0.51 \mathrm{~dB}$ between various distances from laser source. In Fig. 3, we evaluate the transmission loss of our proposed array lens by increasing of the distance from VCSEL array. We find that the transmission loss is $1.9 \mathrm{~dB}$ at $1000 \mathrm{~mm}$ far from VCSEL array. To evaluate the converge-ability of our proposed system, we show that the comparison of the spot size variation with and without our proposed array lens. The spot size of laser source is $360 \mathrm{~nm}$. After our proposed array lens, the spot size is $2000 \mathrm{~nm}$ at $500 \mathrm{~mm}$ far from the VCSEL array. We also find the spot size is still below $2000 \mathrm{~nm}$ at $1000 \mathrm{~mm}$ far from the VCSEL array, too. Therefore, using the array lens, we can increase the irradiation field distribution of VCSEL array in a SMF. For demonstration of our research, we simulated the incoherent irradiance field distribution at $y$-direction with respect to z-axis of the individual microlens array under our arrangements. Fig. 5 shows the irradiance distribution and pattern of the $4 \times 4$ VCSEL array light output after propagating $1000 \mathrm{~mm}$ long of SMF, with via our designed $4 \times 4$ microlens array.

\section{Conclusion}

A $4 \times 4$ nano-scale VCSEL array simultaneously collimated on a SMF has been demonstrated in this paper. Via our proposed $4 \times 4 \mathrm{BK} 7$ microlens array, we can simultaneously conver every laser beams, from $360 \mathrm{~nm}$ to $2000 \mathrm{~nm}$, into a $1000 \mathrm{~mm}$ long SMF. These results show that it is possible to bond multi-elements VCSEL arrays to a SMF, and to simultaneously propagate the entire laser arrays in parallel. Our proposed approach provides design flexibility of the nano-scale spot coupling with the advantages of multi-elements optical power coupling, such as nano-scale VCSEL array. It also can be applied in multi-channels fiber optical communication systems for signal broadcasting to $\mathrm{N} \times \mathrm{N}$ access modes and $\mathrm{N} \times \mathrm{N}$ two dimensional multi-channel output.

Reference

1. Gulden K.H., Brunner M., Eitel S., Gauggel H. P., Hovel R., Hunziker S. And Moser M. 2001 IEEE 
GaAs Digest 53-56.

2. Gudliksen M. S., Lauhon L. J., Wang J., Smith D. C. And Lieber C. M. 2002 Nature 415 617-620.

3. Duan X., Huang Y., Agarwal R. And Lieber C. M. 2003 Nature 421 241-245.

4. Chen F., Zhai J., Stancil D.D., Schlesinger. T. E. 2001 Jp. Appl. Phys. 40 1794-1795.

5. Zemax-EE Optical Design Program, Version 10.0, Nov, 2003.

$4 \times 4$ VCSEL Array

Photo Diode

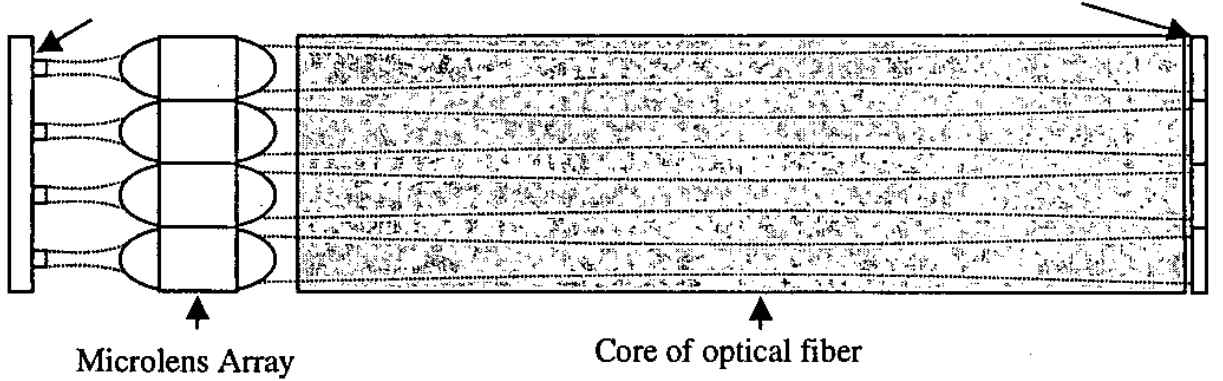

Fig 1. Schematic diagram of the array lens for propagating of 16 elements of VCSEL in a single mode fiber

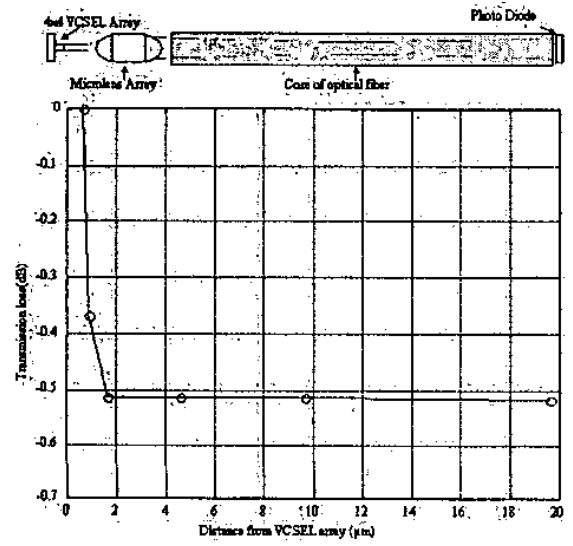

Fig 2. Transmission loss of our proposed array lens, versus various distances from VCSEL is from $0.64 \mu \mathrm{m}$ to $20 \mu \mathrm{m}$

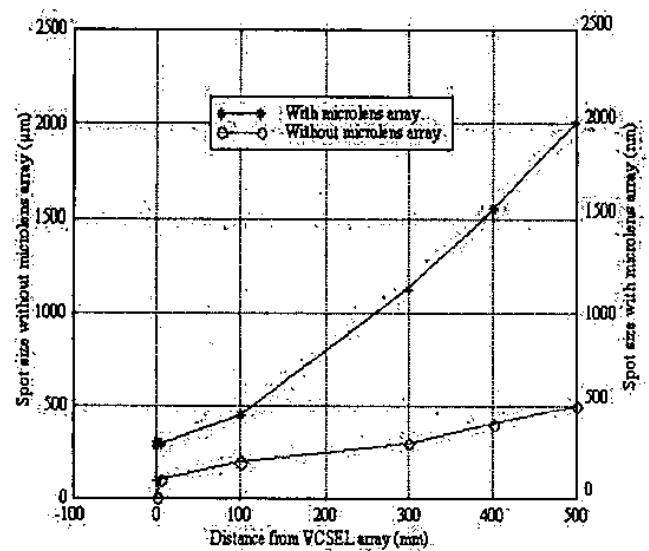

Fig 4. Spot size of VCSEL array, versus various distances from VCSEL array, with and without our proposed array lens

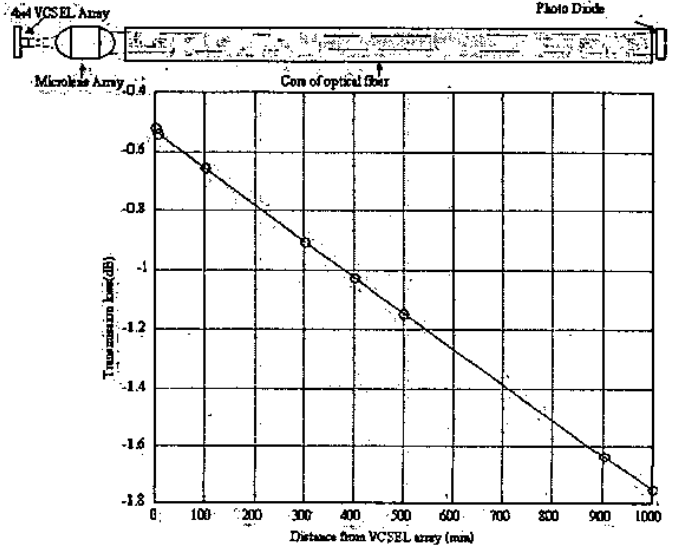

Fig 3. Transmission loss of our proposed array lens, versus various distances from VCSEL is from $20 \mu \mathrm{m}$ to $1000 \mathrm{~mm}$

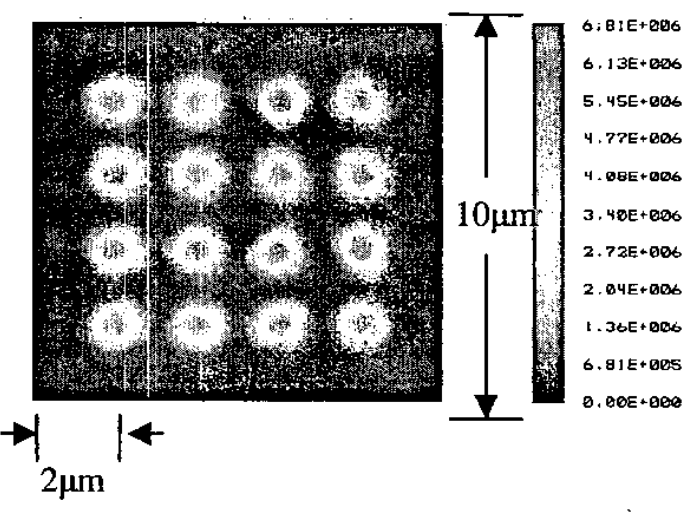

Fig 5. Output optical field intensity of VCSEL array after propagating of $1 \mathrm{~m}$ length of a single mode fiber by our proposed array lens 